\title{
Thalamic and Extrathalamic Mechanisms of Consciousness after Severe Brain Injury
}

\author{
Evan S. Lutkenhoff, PhD, ${ }^{1}$ Jeffrey Chiang, MA, ${ }^{1}$ Luaba Tshibanda, MD, ${ }^{2}$ \\ Evelyn Kamau, MSc, ${ }^{3}$ Murielle Kirsch, MD, ${ }^{2,4}$ John D. Pickard, FMedSci, ${ }^{3}$ \\ Steven Laureys, MD, PhD, ${ }^{2}$ Adrian M. Owen, $\mathrm{PhD}^{5}$ and Martin M. Monti, PhD ${ }^{1,6}$
}

Objective: What mechanisms underlie the loss and recovery of consciousness after severe brain injury? We sought to establish, in the largest cohort of patients with disorders of consciousness (DOC) to date, the link between gold standard clinical measures of awareness and wakefulness, and specific patterns of local brain pathology-thereby possibly providing a mechanistic framework for patient diagnosis, prognosis, and treatment development.

Methods: Structural T1-weighted magnetic resonance images were collected, in a continuous sample of 143 severely brain-injured patients with DOC (and 96 volunteers), across 2 tertiary expert centers. Brain atrophy in subcortical regions (bilateral thalamus, basal ganglia, hippocampus, basal forebrain, and brainstem) was assessed across (1) healthy volunteers and patients, (2) clinical entities (eg, vegetative state, minimally conscious state), (3) clinical measures of consciousness (Coma Recovery Scale-Revised), and (4) injury etiology.

Results: Compared to volunteers, patients exhibited significant atrophy across all structures $(p<0.05$, corrected). Strikingly, we found almost no significant differences across clinical entities. Nonetheless, the clinical measures of awareness and wakefulness upon which differential diagnosis rely were systematically associated with tissue atrophy within thalamic and basal ganglia nuclei, respectively; the basal forebrain was atrophied in proportion to patients' response to sensory stimulation. In addition, nontraumatic injuries exhibited more extensive thalamic atrophy.

Interpretation: These findings provide, for the first time, a grounding in pathology for gold standard behavior-based clinical measures of consciousness, and reframe our current models of DOC by stressing the different links tying thalamic mechanisms to willful behavior and extrathalamic mechanisms to behavioral (and electrocortical) arousal.

ANN NEUROL 2015;00:000-000

$T^{1}$ he mechanisms supporting consciousness, as well as its loss and recovery after severe brain injury, remain largely unknown. In the context of disorders of consciousness $(\mathrm{DOC})^{1}$ such as the vegetative state (VS) and the minimally conscious state (MCS), the lack of a mechanistic understanding of the relationship between brain damage and neurological condition has direct consequences for our ability to make accurate diagnoses, prognoses, and to develop targeted interventions, thereby raising complicated medical and ethical questions. ${ }^{2}$
Although information concerning the nature and extent of a patient's brain damage is generally taken into consideration during clinical assessments, current differential diagnosis procedures rely exclusively —as per international guidelines-on behavioral presentation. ${ }^{3-5}$ Consequently, although our understanding of DOC is continuously increasing, ${ }^{6,7}$ little is known about the connection between behaviorally defined clinical entities and the underlying brain damage, ${ }^{8-10}$ or the degree to which standard behavior-based clinical assessments (eg, JFK

View this article online at wileyonlinelibrary.com. DOI: 10.1002/ana.24423

Received Sep 13, 2014, and in revised form Apr 3, 2015. Accepted for publication Apr 7, 2015.

Address correspondence to Dr Monti, UCLA Department of Psychology, Los Angeles, CA 90095. E-mail: monti@psych.ucla.edu

From the ${ }^{1}$ Department of Psychology, University of California, Los Angeles, Los Angeles, CA; ${ }^{2}$ Coma Science Group, Cyclotron Research Center and Neurology Department, University and University Hospital of Liège, Liège, Belgium; ${ }^{3}$ Division of Neurosurgery, University of Cambridge, Addenbrooke's Hospital, Cambridge, United Kingdom; ${ }^{4}$ Department of Anesthesia and Intensive Care Medicine, Liège University Hospital, Liège, Belgium; ${ }^{5}$ Brain and Mind Institute, University of Western Ontario, London, Ontario, Canada; and ' $B r a i n$ Injury Research Center, Department of Neurosurgery, Geffen School of Medicine at University of California, Los Angeles, Los Angeles, CA 
TABLE 1. Sample Demographic and Groupwise Patient Clinical Information

$\begin{array}{llllllll}\text { Group } & \text { Diagnosis } & \begin{array}{l}\text { Mean Age, } \\ \mathbf{y r}(\mathbf{S D})\end{array} & \begin{array}{l}\text { Gender, } \\ \mathbf{M} / \mathbf{F}\end{array} & \begin{array}{l}\text { Mean NBV, } \\ \mathbf{m m}^{3}(\mathbf{S D})\end{array} & \begin{array}{l}\text { Mean } \\ \text { MPI (SD) }\end{array} & \begin{array}{l}\text { Etiology, } \\ \text { T/NT }\end{array} & \begin{array}{l}\text { Median } \\ \text { CRS-R }\end{array} \\ \begin{array}{l}\mathrm{HV}, \\ \mathrm{n}=96\end{array} & \mathrm{NA} & 33.41(17.65) & 45 / 51 & 1,555,262(134,231.7) & \text { NA } & \text { NA/NA } & \text { NA } \\ \begin{array}{l}\text { Patients, } \\ \mathrm{n}=115\end{array} & \mathrm{VS}, \mathrm{n}=38 & 48.73(18.31) & 23 / 15 & 1,374,257(138,722.3) & 06.41(6.91) & 15 / 23 & 5.5 \\ & & & & & & & \\ & \mathrm{MCS}^{-}, \mathrm{n}=27 & 42.45(16.31) & 16 / 10^{\mathrm{a}} & 1,394,288(136,546.3) & 13.02(13.72) & 18 / 09^{\mathrm{a}} & 9.0 \\ & \mathrm{MCS}^{+}, \mathrm{n}=36 & 43.35(17.02) & 25 / 11 & 1,350,815(107,902.5) & 21.62(30.47) & 23 / 12 & 11.0 \\ & \mathrm{eMCS}, \mathrm{n}=14 & 48.90(19.57) & 10 / 04 & 1,391,304(96,092.88) & 21.15(23.76) & 05 / 09 & 20.5^{\mathrm{b}}\end{array}$

${ }^{\mathrm{a}}$ Unavailable for 1 patient.

${ }^{\mathrm{b} U n a v a i l a b l e ~ f o r ~} 2$ patients.

CRS-R = Coma Recovery Scale-Revised; eMCS = emerging from minimally conscious state; $\mathrm{F}=$ female; $\mathrm{HV}=$ healthy volunteers; $\mathrm{M}=$ male; $\mathrm{MCS}=$ minimally conscious state; $\mathrm{MPI}=$ months postinjury; $\mathrm{NA}=$ not applicable; $\mathrm{NBV}=$ normalized brain volume;

$\mathrm{NT}=$ nontraumatic etiology; $\mathrm{SD}=$ standard deviation; $\mathrm{T}=$ traumatic etiology; $\mathrm{VS}=$ vegetative state.

Coma Recovery Scale-Revised $[\mathrm{CRS}-\mathrm{R}]^{4}$ ) systematically reflect, or index, specific aspects of neural pathology.

In this work, we employ conventional T1-weighted magnetic resonance imaging (MRI) in the largest multicenter continuous sample of DOC patients to date, in an effort to bridge the gap between clinical assessments of consciousness and underlying brain damage. In particular, we attempt to determine a potential minimum common denominator across a characteristically heterogeneous population that might relate standard clinical measures of wakefulness and awareness to specific patterns of subcortical atrophy, thereby providing a mechanistic framework within which to understand loss and recovery of consciousness after severe brain injury, and to develop potential restorative interventions.

As described below, we report a systematic negative association between a patient's quantitative clinical measures of behavioral responsiveness and arousal, and the degree of tissue atrophy within thalamus, basal ganglia, and basal forebrain. These finding provide, for the first time, a clear grounding in pathology for gold standard behaviorbased clinical measures of consciousness, and reframe our current models of DOC by separating the thalamic contribution to willful behavior from the extrathalamic contribution to behavioral (and electrocortical) arousal.

\section{Subjects and Methods}

\section{Participants}

In this cross-sectional study, we recruited a consecutive sample of 143 patients who survived severe brain injury and developed a disorder of consciousness. The sample includes all patients who, between October 2006 and February 2013, underwent structural (T1-weighted) MRI as part of a large multicenter neuroimaging project conducted at the Wolfson Brain Imaging Center, Addenbrooke's Hospital, Cambridge, United Kingdom and the Coma Science Group, University Hospital, University of Liège, Liège, Belgium. The only exclusion criteria were unsuitability for entering the magnetic resonance (MR) environment (eg, any type of non-MR-safe implant) or any acute medical condition making it unsafe for the patient to undergo the procedure (a determination that was made by clinical personnel blinded to the aims of this study). Inclusion criteria were adult patients with acquired severe acute brain injury leading to coma and a subsequent diagnosis of chronic ( $>4$ weeks) DOC (VS or MCS), or emerging from MCS (eMCS), at time of study. As shown in Table 1, about half the patients suffered from a traumatic brain injury, $47 \%$ suffered from nontraumatic brain injury, and the remaining 3\% suffered from a mixed etiology. Almost half of the nontraumatic brain injuries (47\%) were due to anoxic or hypoxic events following cardiac or cardiorespiratory arrest (with only 2 cases reported of hypoglycemic coma and carbon monoxide poisoning). The majority of nontraumatic brain injuries (51\%) were due to cardiovascular events including both ischemic and hemorrhagic stroke. Finally, 1 patient presented with infection (rhombencephalitis).

As described in the data preprocessing section below, 28 patient data sets were discarded because of low image quality (eg, in-scanner motion). Patient exclusion was agreed upon at completion of the initial data preprocessing, prior to any data analysis, and thus blind to the contribution of each of these observations to the overall result. Of the remaining 115 patients (61 from Cambridge and 54 from Liège), 38 met the diagnostic criteria for VS (18 from Cambridge and 20 from Liège), as evaluated with the CRS-R, 63 met the diagnostic criteria for MCS (37 from Cambridge and 26 from Liège), and the 14 remaining met the criteria for eMCS (6 from Cambridge and 8 from Liège; see Table 1). Following the taxonomy recently introduced by Bruno and colleagues, minimally conscious patients were further divided into $27 \mathrm{MCS}^{-}$and $36 \mathrm{MCS}^{+} .{ }^{11}$ 

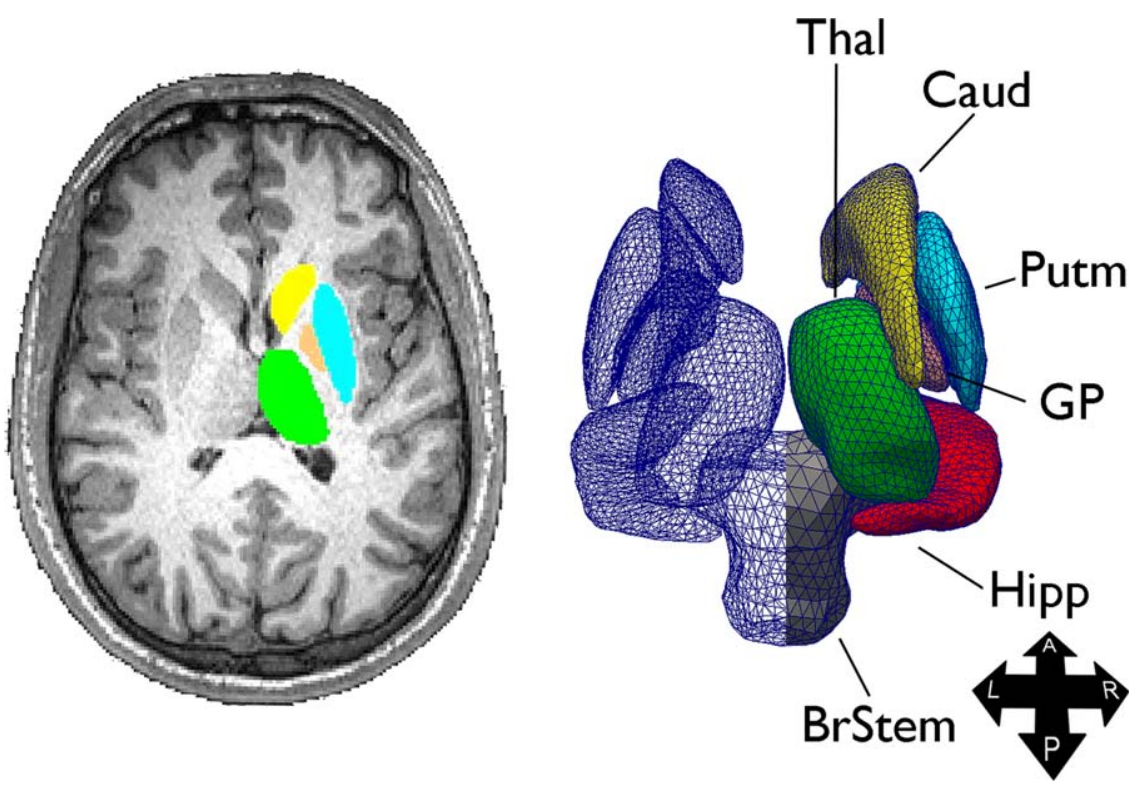

FIGURE 1: Methods. Sample structure extraction (left) and 3-dimensional triangle vertex mesh (right). A $=$ anterior; BrStem = brainstem; Caud = caudate; $G P=$ globus pallidus; Hipp = hippocampus; $L=$ left; $P=$ posterior; Putm $=$ putamen; $\mathrm{R}=$ right; Thal $=$ thalamus.

The study was approved by the Cambridge (UK) Local Research Ethics Committee, and the Ethics Committee of the Medical School of the University of Liège (Belgium). Signed consent was obtained, according to the approved procedures at each site, from each patient's legal surrogate.

Control data were obtained for 96 healthy volunteers (51 female), ranging from 18 to 80 years of age (mean $=33$ years, standard deviation $=18$ years), with no known history of brain disorders (see Table 1). In accordance with the procedure approved by the Cambridge and Liège local ethics research committees, written informed consent was obtained for each volunteer.

\section{Procedures}

Each patient and healthy volunteer underwent a conventional structural T1-weighted 3-dimensional magnetic-preparation rapid gradient echo scan. Patient data were acquired on identical 3T Siemens (Erlangen, Germany) Tim Trio systems at the Wolfson Brain Imaging Centre at Addenbrooke's Hospital (repetition time $[\mathrm{TR}]=2.30$ milliseconds, echo time $[\mathrm{TE}]=2.99$ milliseconds, flip angle $[\mathrm{FA}]=9^{\circ}$, resolution $=1 \times 1 \times 1 \mathrm{~mm}$ ) and at the University Hospital at University of Liège $(\mathrm{TR}=2.30$ milliseconds, $\mathrm{TE}=2.47$ milliseconds, $\mathrm{FA}=9^{\circ}$, resolution $=1 \times 1 \times$ $1.2 \mathrm{~mm})$. Volunteer data were also acquired on a $3 \mathrm{~T}$ Siemens Tim Trio system, at the MRC Cognition and Brain Sciences Unit, Cambridge, United Kingdom ( $\mathrm{TR}=2.30$ milliseconds, $\mathrm{TE}=2.99$ milliseconds, $\mathrm{FA}=9^{\circ}$, resolution $\left.=1 \times 1 \times 1 \mathrm{~mm}\right)$.

\section{Data Preprocessing}

To assess local brain atrophy on the basis of T1-weighted MR images, we employed a technique referred to as "shape (or vertex) analysis,"12 available in FMRIB Software Library (FSL). ${ }^{13}$ Prior to analysis, 3 preprocessing steps were performed. First, data were brain-extracted, using optiBET, ${ }^{14}$ to remove from the images extraneous nonbrain tissue (eg, eyes, neck, skull). Second, subcortical structures of interest were segmented, on an individual basis, and reconstructed into 3-dimensional vertex meshes (as implemented in FSL FIRST). ${ }^{12}$ The segmentation is achieved by incorporating, in a Bayesian framework, individual subject MR image intensities and a set of priors for each target region derived from manual labeling in a set of $336 \mathrm{~T} 1$ weighted MR images. In addition, as part of this process, all data are registered, using a 12 degrees of freedom method, to the nonlinear Montreal Neurological Institute template $(1 \times 1$ $\times 1 \mathrm{~mm}$ resolution), which retains point correspondence between meshes. ${ }^{12}$ Segmentation and mesh construction were performed on all of the following brain regions, separately for each hemisphere: thalamus, caudate nucleus, putamen, globus pallidus, hippocampus, and brainstem (see Fig 1 for an example segmentation and mesh reconstruction). In addition, although not included in the FSL suite, we manually built a segmentation template of the basal forebrain, starting from the probabilistic map made available in the International Consortium for Brain Mapping atlas. ${ }^{15}$ As confirmed visually on the basis of the Atlas of Regional Anatomy of the Brain Using MRI ${ }^{16}$ and the 7.0 Tesla MRI Brain Atlas ${ }^{17}$ (cf pages 83-95 of the latter), the basal forebrain map was mainly centered around the substantia innominata (and thereby the nucleus basalis), as well as the ventral aspect of the septal nucleus, but also overlapped to some extent with the ventral pallidum (ie, the region of the globus pallidus that extends ventrally to the anterior commissure) and the most ventrocaudal segment of the nucleus accumbens. This region was added to the analysis because of its causal role in the maintenance of cortical (and behavioral) arousal in animal models. ${ }^{18}$

Upon completion of this preprocessing step, each participant's segmentations were visually inspected. For 28 patients (13 from Cambridge and 15 from Liège) the segmentation was 


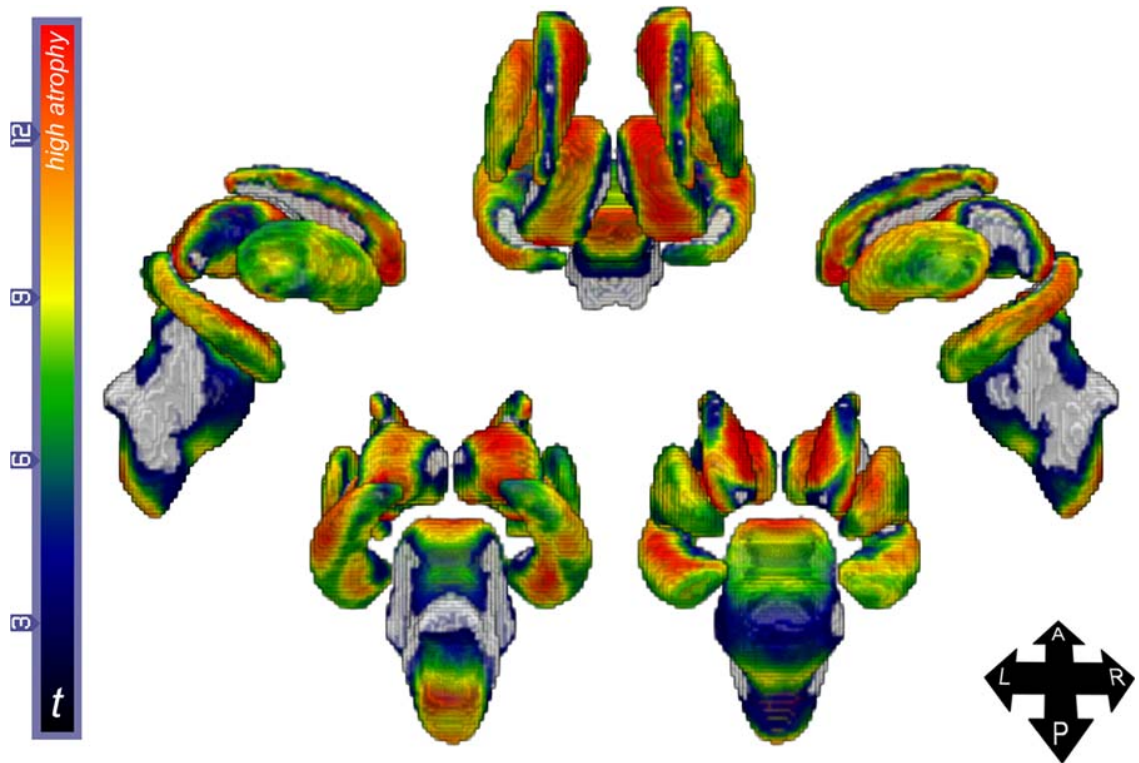

FIGURE 2: Analysis \#1: volunteers versus patients. Colored regions indicate areas of significant atrophy in patients (t statistic), collapsing across diagnoses, as compared to healthy volunteers (warmer colors indicate greater atrophy). Gray areas indicate no significant atrophy. Renderings are in neurological convention. See also Supplementary Video. $A=$ anterior; $L=$ left; $\mathrm{P}=$ posterior; $\mathrm{R}=$ right.

unsuccessful in 1 or more structures, resulting in the exclusion of their data set from the analysis. (We stress that this step took place before any analysis was performed, thus blind to the contribution of each of the 28 excluded observations to the analysis.) Segmentation failure was due to low image quality primarily resulting from in-scanner motion $(n=26)$, and low signal-to-noise ratio presumably due to MR equipment malfunction $(n=2)$. As a last preprocessing step, to account for the effect of head size variability across individuals, we calculated each subject's total normalized brain volume using SIE$\mathrm{NAX}^{19}$ and included this measure as a covariate in all analyses.

\section{Statistical Analysis}

Following preprocessing, the 3-dimensional meshes were entered into 4 separate group analyses. First, to get an overview of the average degree of tissue damage resulting from severe brain injury, we compared healthy volunteers and patients (collapsing across diagnosis; henceforth, analysis \#1). Second, we assessed the relationship between total CRS-R score and local atrophy (analysis \#2). As part of this analysis, we also assessed atrophy differences relating to injury etiology (ie, traumatic vs nontraumatic). Finally, we assessed the relationship between local shape atrophy and CRS-R subscales (ie, auditory function, visual function, motor function, oromotor-verbal, communication, and arousal; analysis \#3). However, because of the correlations between CRS-R subscales, and the negative effects of excessive multicollinearity on regression analysis, ${ }^{20}$ we performed a data reduction (principal component analysis [PCA], with varimax rotation of the loading matrix ${ }^{21}$ ) over the CRS-R subscales. The PCA returned 4 components, which collectively explained $90 \%$ of the total variance. The first component mainly captured the communication and motor function scales (henceforth, Motor-Communication component), the second component mainly isolated auditory and visual scales (Audiovisual component), the third component isolated the arousal scale (Arousal component), and the last component captured the oromotor-verbal scale (Oromotor-Verbal component).

Finally, in a fourth analysis, we investigated differences between clinical groups comparing VS patients to $\mathrm{MCS}^{-}$, $\mathrm{MCS}^{+}$, and eMCS patients, as well as $\mathrm{MCS}^{-}$to $\mathrm{MCS}^{+}$ patients (analysis \#4).

In the first analysis (ie, healthy volunteers vs patients), age, gender, and normalized brain volume were entered in the regression as covariates. In all remaining analyses (ie, all patient-only analyses), time since injury, etiology, and center (ie, Liège, Cambridge) were additionally included as covariates. Significance was established with nonparametric permutation testing against an $\alpha$-criterion of 0.05 corrected for multiple comparisons using a familywise cluster correction using FSL Randomise module. ${ }^{22,23}$

\section{Results}

Compared to healthy volunteers (ie, analysis \#1), patients exhibited significant atrophy across all examined regions.

As depicted in Figure 2 (and Supplementary Video), extensive atrophy was detected in globus pallidus (100\% and $99 \%$ of vertices in the right and left meshes, respectively), putamen ( $96 \%$ and $94 \%$ of vertices), hippocampus ( $93 \%$ and $95 \%$ of vertices), thalamus ( $97 \%$ and $88 \%$ of vertices). Extensive atrophy, albeit in fewer vertices, was also detected in the caudate nucleus $(80 \%$ and $82 \%$ of vertices in the right and left meshes, respectively), brainstem (73\% of vertices), and basal forebrain (53\% and $55 \%$ of vertices). Within these regions, peaks 
A CRS-R Total
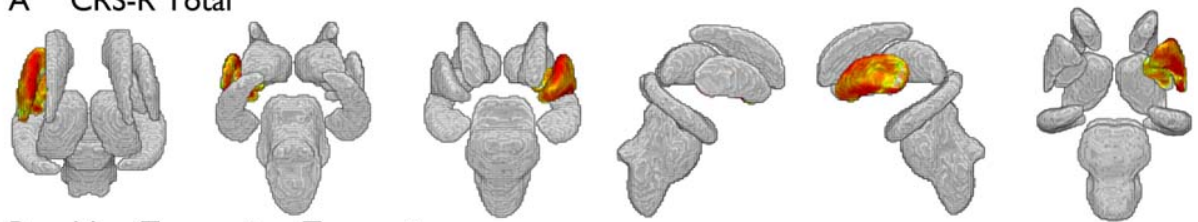

B Non-Traumatic $v$ Traumatic
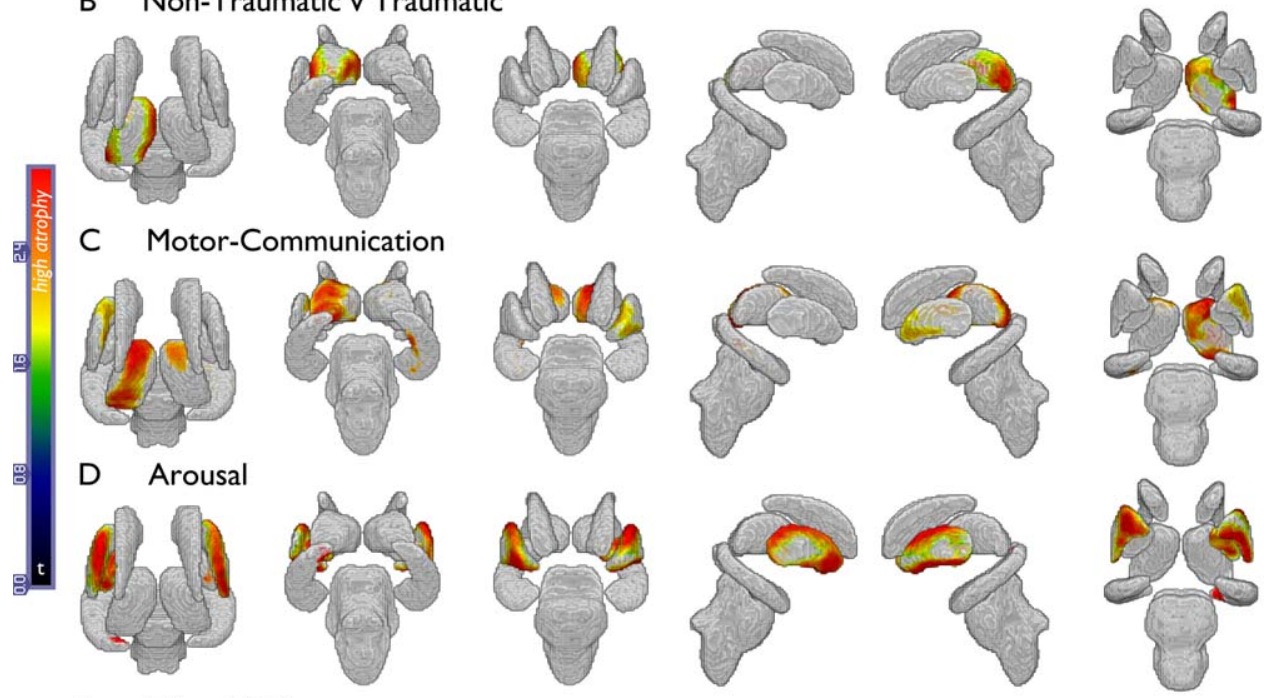

E VS v eMCS

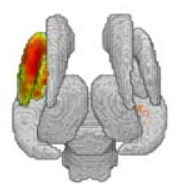

Dorsal

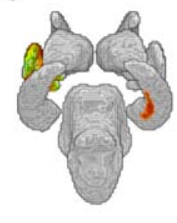

Posterior

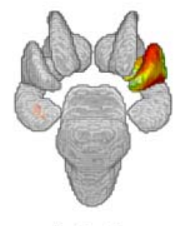

Anterior

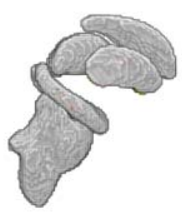

Right

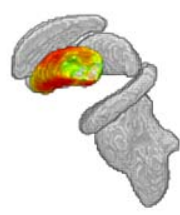

Left

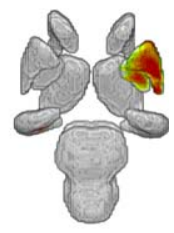

Ventral

FIGURE 3: Analyses \#2, \#3, and \#4. (A) Regions (negatively) correlating with total Coma Recovery Scale-Revised (CRS-R) score. (B) Regions of greater atrophy in patients suffering from nontraumatic brain injury, as compared to patients with traumatic brain injury. (C) Regions (negatively) correlating with the communication-motor subscale component. (D) Regions (negatively) correlating with the arousal subscale component. (E) Regions of greater atrophy in vegetative state (VS) patients as compared to emerging from minimally conscious state (eMCS) patients. Gray areas indicate no significant atrophy.

of maximal atrophy occurred in bilateral anterodorsal thalami, anterior dorsomedial caudate nuclei, and anterior hippocampus.

As shown in Figure 3A, the CRS-R total score (analysis \#2) correlated inversely with atrophy in large portions of the left globus pallidus (76\% of vertices), left putamen ( $85 \%$ of vertices), and small sections of the bilateral ventromedial basal forebrain (8\% and 2\% of right and left vertices, respectively; Fig 4C). In addition, after having factored out the total clinical score (as well as all the covariates described in the Subjects and Methods section), nontraumatic injury was still associated with increased atrophy within the lateral and medial sections of left thalamus ( $56 \%$ of vertices), as well as a very small section of the left basal forebrain ( $1 \%$ of vertices), as compared to traumatic brain injury (see Fig 3B). When we followed up this result with a comparison within nontraumatic brain injury patients (ie, anoxic/hypoxic versus cardiovascular etiologies), no significant differences were observed.
When assessing the individual PCA components derived over the CRS-R subscores (analysis \#3), the Motor-Communication component scores exhibited a significant negative correlation with the degree of atrophy in bilateral anterior and dorsomedial thalamus (61\% and $27 \%$ of vertices in the left and right hemisphere, respectively), small segments of left putamen (36\% of vertices), and right medial and posterolateral hippocampus (25\% of vertices; see Fig 3C). The Arousal component was negatively associated with extensive atrophy in bilateral putamen $(69 \%$ and $63 \%$ in left and right hemisphere, respectively), globus pallidus (92\% and $16 \%$ of vertices), and a small segment of left posterior hippocampus (3\% of vertices; see Fig 3D). The Audiovisual component was negatively associated with the degree of atrophy in small bilateral ventromedial segments of the basal forebrain $(3 \%$ and $5 \%$ of vertices in the left and right hemisphere, respectively; see Fig 4D). No significant associations were observed between the Oromotor- 
A Basal Forebrain ROI

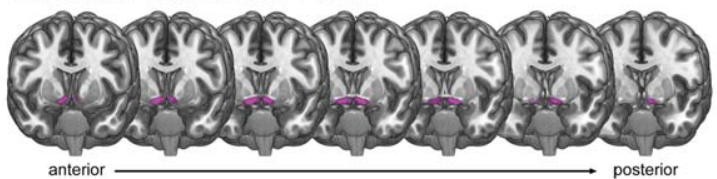

B Patients v. Volunteers

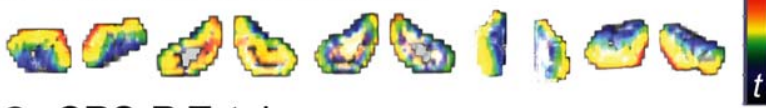

C CRS-R Total

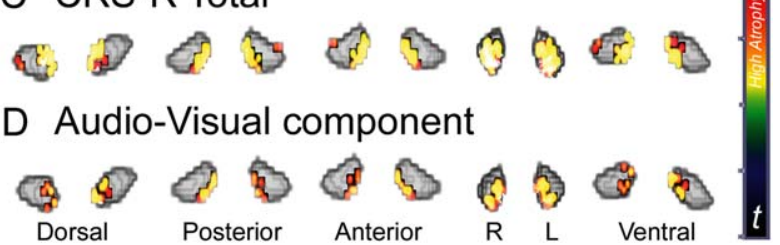

FIGURE 4: Basal forebrain. (A) Basal forebrain standard region of interest (ROI) overlaid on top of a standard highresolution healthy brain. (B) Results for the healthy volunteers versus patients comparison (analysis \#1). (C) Basal forebrain subregions (negatively) correlating with total Coma Recovery Scale-Revised (CRS-R) (analysis \#2). (D) Basal forebrain subregions (negatively) correlating with Audiovisual subscales component (analysis \#3). Note that the $t$ statistic color schema for analysis \#1 ranges from 0 to 12, whereas for analysis \#2 and \#3 it ranges from 0 to 2.8 .

Verbal component and local shape change in any of the target regions.

Finally, comparison across patient groups (analysis \#4) resulted in very few observable differences. No significant differences were detected in the comparison of VS versus $\mathrm{MCS}^{-}$patients, VS versus $\mathrm{MCS}^{+}$patients, and $\mathrm{MCS}^{-}$versus $\mathrm{MCS}^{+}$patients. The only significant difference across clinical entities was observed when comparing the extremes of the DOC spectrum, VS and eMCS patients (see Fig 3E). Consistent with the result reported for total CRS-R, VS patients exhibited, as compared to eMCS, significant atrophy in left putamen ( $85 \%$ of vertices) and globus pallidus (69\% of vertices), as well as in small sections in medial and anterior right hippocampus (6\% of vertices). This result was obtained with fewer observations (ie, 38 vs 14) than those available for the remaining group comparisons, thus suggesting that the null results we reported when comparing VS to $\mathrm{MCS}^{+/-}$ patients (which parallel a previous small sample study ${ }^{8}$ ) might not just be a consequence of low power. (No vertices appeared significant in the reverse of any of the above comparisons; eg, in no region did patients exhibit expansion as compared to healthy volunteers, or positive correlations with any of the continuous variables.)

\section{Discussion}

In this study, we have reported 3 main findings. First, we have shown, for the first time and in the largest sample to date, that DOC patients exhibit extensive atrophy across a number of subcortical structures including regions known to be involved in the regulation of electrocortical arousal, sleep-wake rhythms, and conscious behavior. ${ }^{24-27}$ Second, we have shown that the graded clinical measures upon which diagnostic stratification rely (ie, the CRS-R subscales) reflect systematic "minimum common" brain pathology, as detected with MRI, in relation to specific aspects of consciousness (as clinically defined). ${ }^{28}$ On the one hand, the negative association between motor/communication scores and atrophy along the anterior and dorsomedial regions of thalamus is consistent with the mesocircuit theory according to which corticopetal projections from thalamus to prefrontal cortex are crucial for sustaining organized behavior ${ }^{26}$ and integrating information across different regions of cortex. ${ }^{29}$ These regions are known to be a target of secondary, nonmechanic, damage (eg, Wallerian degeneration) in acute moderate-to-severe brain injury, with the degree of atrophy correlating with long-term outcome. ${ }^{10}$ Furthermore, the dorsomedial aspect of thalamus is known to be the main subcortical structure projecting to prefrontal cortex, ${ }^{30}$ and to play a key role in the regulation of higher cognitive functions in DOC patients. ${ }^{31}$ Stimulation of neurons in this area has been shown to lead to increased responsiveness in some MCS patients. ${ }^{27}$ On the other hand, our data show that arousal-as measured by the CRS-R-is inversely correlated with the degree of atrophy in bilateral basal ganglia. This aspect of our finding is in keeping with a growing literature suggesting that the basal ganglia serve a critical role in the maintenance of behavioral and electrocortical arousal, as well as wakefulness (as supported by the many arousal and sleep/wake components in striatal dysfunction syndromes). ${ }^{24,25,32-34}$ Lesions to the dorsal striatum have been shown in animal models to result in a reduction in total wake, wake fragmentation, and greater power in the electroencephalographic (EEG) $\delta$ band $(0.5-4 \mathrm{~Hz})$, the latter of which is observed across behavioral states, ${ }^{33}$ and matches a pattern that is often observed in DOC patients. ${ }^{35}$ Furthermore, computational models have recently shown that, under conditions of decreased $\gamma$ aminobutyric acidergic (GABAergic) input from the striatum to the globus pallidus pars externa (a circuit that our data suggest is pathological in DOC patients, at the globus pallidus end), oscillations in the $\beta$ frequency $(15-30 \mathrm{~Hz})$ emerge spontaneously, ${ }^{36}$ which is another feature of DOC pathophysiology. ${ }^{35}$ More surprising is the absence of association between patients' level of arousal and thalamic atrophy. Thalamic intralaminar and midline nuclei are traditionally considered to be a key element of the ascending reticular system, crucial for electrocortical and behavioral arousal. ${ }^{37}$ Nonetheless, in animal models, 
complete cell-body-specific lesions in thalamus that spare fibers of passage (as well as cell-body-specific lesions in the globus pallidus pars interna, another nucleus considered to be part of the mesocircuit underlying disorders of consciousness $^{35}$ ) have been shown to produce little effect on behavioral measures of wakefulness, EEG rhythms in frontoparietal regions, and activation of cortical neurons during wakefulness and stimulation, ${ }^{18,38,39}$ even when specifically focused on the intralaminar nuclei. ${ }^{40}$ Conversely, cell-body-specific lesions within the basal ganglia (eg, caudate and putamen, globus pallidus pars externa) have each been shown to cause a slowing of the EEG toward greater $\delta$ and lower $\theta$ density, despite an intact thalamus. ${ }^{33}$ Although it should be recognized that important differences might exist between the examined animal models and the human brain, our findings are consistent with the above literature and do support the view that "while thalamus might be crucial for transmitting specific information that provides the content of the waking state, and may therefore contribute to overall arousal if thalamic input demands attention, it might itself be neither necessary nor sufficient to produce wakefulness." ${ }^{18}$ At a circuit level, the implication of our findings is that, at least in the context of DOC, different aspects of consciousness, including willful behavior and arousal, might be mediated by thalamic and extrathalamic mechanisms, respectively. On the one hand, a corticostriatopallido(internal)-thalamocortical mesocircuit might be crucial for maintaining large-scale, organized, willful behavior. ${ }^{7,26}$ On the other hand, electrocortical and behavioral arousal might be principally sustained by extrathalamic circuits including a corticostriatopallido(external)-cortical circuit, ${ }^{24,25,33,34,41-43}$ which would directly explain our findings, as well as the (glutamatergic) innervations emanating from the parabrachial/precoeruleus complex, which has been previously shown to be crucial for this process (but could not be assessed with the present technique). ${ }^{18,44}$ In addition, we also found that the basal forebrain was (negatively) associated with the degree of sensory responsiveness of patients. This region is well known to play a causal role in the maintenance of behavioral and electrocortical arousal, and to induce a comalike state when extensively lesioned. ${ }^{18}$ Nonetheless, the basal forebrain has also been shown to be important for fast cortical modulation aimed at transiently amplifying cortical activity and the processing of sensory stimuli (putatively via the inhibitory action of basal forebrain GABAergic projections on inhibitory cortical interneurons). ${ }^{45-48}$ One might therefore speculate that the degree of atrophy we observed in this region ( $~ 50 \%$ of the region of interest, as compared to healthy volunteers) might not have been sufficient to induce the comalike state seen in animal models upon complete lesion of this region, ${ }^{18}$ but might nonetheless have been sufficient to affect the mechanisms of fast cortical disinhibition. Future studies will have to address this possibility, as well as the exact relationship between the acute brainstem and basal forebrain comalike state observed in animal models, ${ }^{18,44}$ acute brainstem coma in humans, ${ }^{49}$ and chronic disorders of consciousness where some level of spontaneous arousal is recovered, in the absence of (self-)awareness.

Third, paralleling postmortem examinations, ${ }^{50}$ we have also shown in vivo that patients suffering from nontraumatic brain injury exhibit a more widespread and left-lateralized degree of thalamic atrophy, consistent with the well-known poorer prognosis associated with nontraumatic etiology. ${ }^{1}$ We note that the left lateralization of the additional atrophy does not appear to be a trivial consequence of greater prevalence of left-lateralized injuries in our sample, which according to the clinical notes were mostly bilateral $(84 \%$ vs $9.6 \%$ and $6 \%$ of left- and right-lateralized injuries, respectively). The functional significance of this lateralization remains to be evaluated. Nonetheless, this finding further highlights the dependence of clinical (and neuroimaging-based) assessments of consciousness on residual linguistic processing, and the necessity to develop non-language-based procedures for eliciting willful (motor or neural) responses, ${ }^{51,52}$ as well as non-response-dependent functional ${ }^{29,53,54}$ and structural $^{8-10}$ biomarkers of consciousness. On a similar note, it is important to stress that this work has exclusively focused on the clinical interpretation of the cardinal elements of consciousness (ie, presence of nonreflexive responsiveness and eye opening), ${ }^{3,4,55}$ and did not address the more ephemeral subjective/phenomenological aspect of human consciousness.

In conclusion, our data differentiate the link between thalamic damage and goal-directed, willful behavior $^{6}$ on the one hand, and extrathalamic damage and behavioral (and cortical) arousal on the other. It is noteworthy, however, that diagnostic groups (eg, VS, MCS) do not appear to be readily distinguishable in terms of subcortical structural pathology, presumably due to extensive within-category variance. These findings reframe our current understanding of the brain pathology underlying loss and recovery of consciousness, and provide, for the first time, a direct link between behaviorally based clinical measures of consciousness and "minimum common" localized brain injury, thereby also informing our search for restorative therapeutic interventions.

Finally, interpretation of our findings should be mindful of a number of limitations. First, our measure of atrophy is actually a measure of local tissue 
displacement, and is therefore unable to differentiate the contribution of different cell populations within the regions we assessed to the overall effect. Furthermore, the use of structural neuroimaging, a descriptive methodology, to pinpoint neuroanatomical components of consciousness, as measured with standard clinical methods, does not allow a causal interpretation of our findings. Second, the atlas-based component of our approach is both an asset, allowing improved estimation and segmentation of individual regions, and a liability, given its reliance on priors estimated mostly from healthy volunteers. Third, it should also be noted that our proposal, as well as the mesocircuit model of DOC, ${ }^{7}$ are to be understood as approximations that do not take into full account the extent of corticosubcortical connectivity, its temporal dynamics, ${ }^{56}$ the multiple mechanisms of action of neurotransmitters, and a number of additional regions known to be important for the regulation of arousal and cortical function. ${ }^{34}$ Finally, we also note that we have focused on the idea of a "minimum common denominator" across a heterogeneous sample of patients, employing a multiple regression approach to control for a number of factors that are known to be important sources of variability in DOC. Nonetheless, it must be recognized that there are a number of additional factors that might well play an important role in determining the specific pattern of atrophy observed in a given patient that we could not address (eg, whether pharmacologic coma was used, the presence of status epilepticus, the potential of some patients undergoing steroid administration) and that will likely require even larger samples than the present one to allow full statistical evaluation.

\section{Acknowledgment}

This study was supported by the James S. McDonnell Foundation "Scholar Award" (M.M.M.), the Medical Research Council UK (U.1055.01.002.00007.01, U.1055.01.002.00001.01; A.M.O.), a UK National Institute for Health Research Senior Investigator Award (J.D.P.), the Belgian National Funds for Scientific Research (FRS-FNRS; S.L.), the European Commission ICT Program Project FP7-247919 (A.M.O., S.L.), and the Canada Excellence Research Chairs Program (A.M.O.).

\section{Authorship}

M.M.M. conceived the study. M.M.M., E.K., L.T., and M.K., acquired the data. E.S.L. and J.C. performed imaging preprocessing. E.S.L. conducted the data analysis. M.M.M. and E.S.L. interpreted the data. M.M.M. and E.S.L. drafted the manuscript. All authors were involved in subsequent revisions. M.M.M., A.M.O., S.L., and J.D.P. secured the funding.

\section{Potential Conflicts of Interest}

Nothing to report.

\section{References}

1. Monti MM, Laureys S, Owen AM. The vegetative state. BMJ 2010; 341:c3765

2. Jennett $B$. Thirty years of the vegetative state: clinical, ethical and legal problems. Prog Brain Res 2005;150:537-543.

3. Giacino JT, Ashwal S, Childs N, et al. The minimally conscious state: definition and diagnostic criteria. Neurology 2002;58:349353.

4. Giacino JT, Kalmar K, Whyte J. The JFK Coma Recovery ScaleRevised: measurement characteristics and diagnostic utility. Arch Phys Med Rehabil 2004;85:2020-2029.

5. Multi-Society Task Force on PVS. Medical aspects of the persistent vegetative state (1). N Engl J Med 1994;330:1499-1508.

6. Schiff ND. Central thalamic contributions to arousal regulation and neurological disorders of consciousness. Ann N Y Acad Sci 2008; 1129:105-118.

7. Schiff ND. Recovery of consciousness after brain injury: a mesocircuit hypothesis. Trends Neurosci 2010;33:1-9.

8. Fernández-Espejo D, Junque $C$, Bernabeu $M$, et al. Reductions of thalamic volume and regional shape changes in the vegetative and the minimally conscious states. J Neurotrauma 2010;27:11871193.

9. Fernández-Espejo D, Bekinschtein T, Monti MM, et al. Diffusion weighted imaging distinguishes the vegetative state from the minimally conscious state. Neuroimage 2011;54:103-112.

10. Lutkenhoff ES, McArthur DL, Hua X, et al. Thalamic atrophy in antero-medial and dorsal nuclei correlates with six-month outcome after severe brain injury. Neuroimage Clin 2013;3:396-404.

11. Bruno MA, Vanhaudenhuyse A, Thibaut $A$, et al. From unresponsive wakefulness to minimally conscious PLUS and functional locked-in syndromes: recent advances in our understanding of disorders of consciousness. J Neurol 2011;258:1373-1384.

12. Patenaude B, Smith SM, Kennedy DN, Jenkinson M. A Bayesian model of shape and appearance for subcortical brain segmentation. Neuroimage 2011;56:907-922.

13. Smith SM, Jenkinson M, Woolrich MW, et al. Advances in functional and structural MR image analysis and implementation as FSL. Neuroimage 2004;23(suppl 1):S208-S219.

14. Lutkenhoff ES, Rosenberg M, Chiang J, et al. Optimized brain extraction for pathological brains (optiBET). PloS One 2014;9: e115551.

15. Mazziotta J, Toga A, Evans A, et al. A probabilistic atlas and reference system for the human brain: International Consortium for Brain Mapping (ICBM). Philos Trans R Soc Lond B Biol Sci 2001; 356:1293-1322.

16. Tamraz JC, Comair YG, Tamraz J. Atlas of regional anatomy of the brain using MRI. New York, NY: Springer, 2004.

17. Cho Z-H. 7.0 Tesla MRI brain atlas: in vivo atlas with cryomacrotome correlation. New York, NY: Springer, 2010.

18. Fuller PM, Sherman D, Pedersen NP, et al. Reassessment of the structural basis of the ascending arousal system. J Comp Neurol 2011;519:933-956. 
19. Smith SM, Zhang $Y$, Jenkinson $M$, et al. Accurate, robust, and automated longitudinal and cross-sectional brain change analysis. Neuroimage 2002;17:479-489.

20. Monti MM. Statistical analysis of $\mathrm{fMRI}$ time-series: a critical review of the GLM approach. Front Hum Neurosci 2011;5:28.

21. Kaiser HF. The varimax criterion for analytic rotation in factor-analysis. Psychometrika 1958;23:187-200.

22. Smith SM, Nichols TE. Threshold-free cluster enhancement: addressing problems of smoothing, threshold dependence and localisation in cluster inference. Neuroimage 2009;44:83-98.

23. Winkler AM, Ridgway GR, Webster MA, et al. Permutation inference for the general linear model. Neuroimage 2014;92:381-397.

24. Lazarus M, Chen JF, Urade Y, Huang ZL. Role of the basal ganglia in the control of sleep and wakefulness. Curr Opin Neurobiol 2013:23:780-785.

25. Lazarus M, Huang Z-L, Lu J, et al. How do the basal ganglia regulate sleep-wake behavior? Trends Neurosci 2012;35:723-732.

26. Schiff ND. Central thalamic contributions to arousal regulation and neurological disorders of consciousness. Ann N Y Acad Sci 2008;1129:105-118.

27. Schiff ND, Giacino JT, Kalmar K, et al. Behavioural improvements with thalamic stimulation after severe traumatic brain injury. Nature 2007:448:600-603.

28. Laureys S. The neural correlate of (un)awareness: lessons from the vegetative state. Trends Cogn Sci 2005;9:556-559.

29. Monti MM, Lutkenhoff ES, Rubinov M, et al. Dynamic change of global and local information processing in propofol-induced loss and recovery of consciousness. PLoS Comput Biol 2013;9: e1003271.

30. Klein JC, Rushworth MF, Behrens TE, et al. Topography of connections between human prefrontal cortex and mediodorsal thalamus studied with diffusion tractography. Neuroimage 2010;51: $555-564$.

31. Monti MM, Rosenberg M, Finoia $\mathrm{P}$, et al. Thalamo-frontal connectivity mediates top-down cognitive functions in disorders of consciousness. Neurology 2015;84:167-173.

32. Magill PJ, Bolam JP, Bevan MD. Relationship of activity in the subthalamic nucleus-globus pallidus network to cortical electroencephalogram. J Neurosci 2000;20:820-833.

33. Qiu MH, Vetrivelan R, Fuller PM, Lu J. Basal ganglia control of sleep-wake behavior and cortical activation. Eur J Neurosci 2010; 31:499-507.

34. Vetrivelan R, Qiu MH, Chang C, Lu J. Role of basal ganglia in sleep-wake regulation: neural circuitry and clinical significance. Front Neuroanat 2010;4:145

35. Schiff ND, Nauvel T, Victor JD. Large-scale brain dynamics in disorders of consciousness. Curr Opin Neurobiol 2014;25:7-14.

36. Holgado AJ, Terry JR, Bogacz R. Conditions for the generation of beta oscillations in the subthalamic nucleus-globus pallidus network. J Neurosci 2010;30:12340-12352.

37. Van der Werf YD, Witter MP, Groenewegen HJ. The intralaminar and midline nuclei of the thalamus. Anatomical and functional evidence for participation in processes of arousal and awareness. Brain Res Brain Res Rev 2002;39:107-140.
38. Pritzel $M$, Markowitsch HJ. Kainic acid lesions in the cat's thalamus: morphological and behavioral changes. Brain Res Bull 1980; 5:61-67.

39. Vanderwolf $\mathrm{CH}$, Stewart DJ. Thalamic control of neocortical activation: a critical re-evaluation. Brain Res Bull 1988;20:529-538.

40. Constantinople CM, Bruno RM. Effects and mechanisms of wakefulness on local cortical networks. Neuron 2011;69:1061-1068.

41. Gritti I, Mainville L, Mancia M, Jones BE. GABAergic and other noncholinergic basal forebrain neurons, together with cholinergic neurons, project to the mesocortex and isocortex in the rat. J Comp Neurol 1997;383:163-177.

42. Qiu MH, Chen MC, Huang ZL, Lu J. Neuronal activity (c-Fos) delineating interactions of the cerebral cortex and basal ganglia. Front Neuroanat 2014;8:13.

43. Gritti I, Manns ID, Mainville L, Jones BE. Parvalbumin, calbindin or calretinin in cortically projecting and GABAergic, cholinergic, or glutamatergic basal forebrain neurons of the rat. J Comp Neurol 2003;458:11-31.

44. Kaur S, Pedersen NP, Yokota S, et al. Glutamatergic signaling from the parabrachial nucleus plays a critical role in hypercapnic arousal. J Neurosci 2013;33:7627-7640.

45. Avila I, Lin S-C. Motivational salience signal in the basal forebrain is coupled with faster and more precise decision speed. PLoS Biol 2014;12:e1001811.

46. Lin S-C, Gervasoni D, Nicolelis MA. Fast modulation of prefrontal cortex activity by basal forebrain noncholinergic neuronal ensembles. J Neurophysiol 2006;96:3209-3219.

47. Goard M, Dan Y. Basal forebrain activation enhances cortical coding of natural scenes. Nat Neurosci 2009;12:1444-1449.

48. Nguyen DP, Lin S-C. A frontal cortex event-related potential driven by the basal forebrain. Elife 2014;3:e02148.

49. Parvizi J, Damasio AR. Neuroanatomical correlates of brainstem coma. Brain 2003;126:1524-1536.

50. Adams JH, Graham DI, Jennett B. The neuropathology of the vegetative state after an acute brain insult. Brain 2000;123(pt 7): $1327-1338$

51. Bekinschtein TA, Shalom DE, Forcato $C$, et al. Classical conditioning in the vegetative and minimally conscious state. Nat Neurosci 2009:12:1343-1349.

52. Bekinschtein TA, Dehaene S, Rohaut B, et al. Neural signature of the conscious processing of auditory regularities. Proc Natl Acad Sci U S A 2009:106:1672-1677.

53. Crone JS, Schurz M, Höller $Y$, et al. Impaired consciousness is linked to changes in effective connectivity of the posterior cingulate cortex within the default mode network. Neuroimage 2015; 110:9.

54. Chennu S, Finoia P, Kamau E, et al. Spectral signatures of reorganised brain networks in disorders of consciousness. PLoS Comput Biol 2014;10:e1003887.

55. Monti MM, Owen AM. Behavior in the brain using functional neuroimaging to assess residual cognition and awareness after severe brain injury. J Psychophysiol 2010;24:76-82.

56. Jaeger D, Kita H. Functional connectivity and integrative properties of globus pallidus neurons. Neuroscience 2011;198:44-53. 